\title{
EL HIJO SUSTITUIDO: PROYECCIONES DE UN MOTIVO MÍTICO Y FOLKLÓRICO EN LA LITERATURA Y EL CINE
}

\section{The Substituted Child: Projections of a Mythical and Folkloric Motif in Literature and Cinema}

\author{
Mercedes AgUIRRE CASTRO \\ macics@yaboo.co.uk
}

Recibido: 14/10/2020; Aceptado: 02/12/2020; Publicado: 31/12/2021

Ref. Bibl. MERCEDES AGUIRRE CASTRO. EL HIJO SUSTITUIDO: PROYECCIONES DE UN MOTIVO MÍTICO Y FOLKLÓRICO EN LA LITERATURA Y EL CINE. 1616: Anuario de Literatura Comparada, 11 (2021), 163-180

RESUMEN: Este artículo examina el motivo del hijo sustituido, desde la mitología clásica y las leyendas relativas al mundo de las hadas, pasando después a analizar algunos ejemplos literarios en diferentes momentos históricos y, finalmente, ver la presencia de este tema en el cine actual. El artículo trata de ver de qué manera se puede identificar una continuidad de este motivo en unas historias que, a primera vista, no parecen tener nada en común, pero que de alguna forma dejan translucir temáticas que proceden de los antiguos mitos y creencias aunque con las diferencias lógicas teniendo en cuenta las circunstancias sociales de las épocas en las que los motivos aparecen. En cualquier caso, lo que coincide en dichos ejemplos es que responden a actitudes hacia unos conflictos que se relacionan con la maternidad.

Palabras clave: changeling; sustitución; niños; mito; folklore; maternidad; literatura; cine. 
ABSTRACT: This article examines the motif of the substituted child, beginning with Classical mythology and legends about the fairy world, continuing through some literary examples from different historical moments, and finally the presence of this theme in some modern films. The article aims to examine how a continuity of this motif can be identified in a series of stories which, in the first instance, seem to have nothing in common, but which in some way reveal the presence of themes from ancient myths and beliefs, although with differences corresponding to social circumstances at the times when these themes appear. In any case, what runs through all of these examples is a concern with conflicts relating to maternity.

Key words: changeling; substitution; children; myth; folklore; maternity; literature; cinema.

Cuando Shakespeare en El sueño de una noche de verano ${ }^{1}$ se refiere a que un niño es un changeling está aludiendo a un tema y a una creencia bien conocida en su época: que los niños podían ser sustituidos por las hadas, que, en su lugar, dejaban a sus propios hijos. Este es quizá el ejemplo más conocido en la literatura occidental sobre el motivo de la sustitución de un niño, pero lo que es menos conocido es que en realidad este tema forma parte de un panorama cultural y literario más amplio y, para entenderlo, es necesario contemplarlo primero hacia atrás y luego hacia adelante en el tiempo.

Tomando, pues, como punto de partida una selección de mitos clásicos y sus fuentes literarias y un material procedente del folklore ${ }^{2}$, este artículo examina el motivo general del hijo sustituido y el llamado changeling que la literatura ha usado y reelaborado en diferentes momentos históricos e, incluso, emerge en el cine contemporáneo. El material está presentado cronológicamente, comenzando con algunos ejemplos en la mitología griega y las fuentes literarias en las que dichos mitos están transmitidos, pasando después a las creencias populares en torno al mundo de las hadas y su reflejo en la literatura y, posteriormente, en la ficción cinematográfica. De

1. II. 1.23 .

2. Una posible definición de folklore sería considerarlo como parte tradicional de la cultura de un pueblo que se ha preservado, consciente o inconscientemente, en creencias, prácticas y costumbres. Ambos, mito y folklore, podrían coincidir en su transmisión oral, pero es a menudo difícil separar lo que pertenece al ámbito del mito y lo que pertenece más propiamente al folklore. Se podría citar una bibliografía extensísima sobre el tema, pero este no sería el lugar para investigarlo. En cualquier caso, mi argumento no se vería afectado por la cuestión de encontrar una definición. 
esta forma, teniendo en cuenta las diferencias que lógicamente emergen de las circunstancias sociales de las épocas en las que los motivos aparecen, trataremos de ver de qué manera existe una continuidad de este tema en unas historias y relatos que a primera vista no parecen tener nada en común, pero que, de alguna forma, dejan translucir temáticas que proceden de los antiguos mitos y creencias y que responden a actitudes hacia unos conflictos que se relacionan con la maternidad y el miedo que rodea al momento del nacimiento de un hijo y su aceptación por la comunidad.

La idea de un sustituto de un niño que pretende pasar por el auténtico la encontramos ya en el mito griego de Crono y $\mathrm{Rea}^{3}$, que es uno de los ejemplos más tempranos de un motivo general de "sustitución» que aparece en una variedad de formas en la mitología griega (Davidson 1995, 363). En el relato hesiódico Rea entrega a Crono una piedra envuelta en pañales -es decir, con la apariencia de un niño recién nacido- en lugar de su último hijo, que este traga como ha hecho ya con su anterior progenie para impedir ser destronado por uno de sus hijos (Versnel 1987, 122-123). No me voy a detener aquí en los orígenes míticos y cultuales de esta piedra ${ }^{4}$, sino en el hecho de que una vez vestida resulta del suficiente tamaño y peso para ser confundida con el pequeño y por lo tanto ser devorada por Crono, asumiendo que este no se percata de la inmovilidad de la piedra. Precisamente por su inmovilidad, falta de vida y voz y, a la vez, por sus vestidos, la piedra se transforma aquí en un muñeco que cobra vida en la mente de Crono al tomarlo por su hijo. La piedra se convierte en un «doble» del niño auténtico5. Davidson $(1995,368)$ señala acertadamente que el truco no habría servido con Rea: a una madre no la habrían engañado si le hubieran ofrecido una alternativa del niño.

La sustitución es realizada, por tanto, por la madre y para proteger al niño auténtico, Zeus, que será escondido y criado en Creta. Versiones latinas ofrecen más detalles de esta parte de la historia: así Ovidio presenta a las ninfas criando al bebé Zeus con la leche de la cabra Amaltea ${ }^{6}$ (Davidson 1995, 365). La versión de Servio ${ }^{7}$ dice que fueron abejas las que criaron al niño en Creta (Davidson 1995, 367).

La mitología griega ofrece asimismo una historia en la que aparece, si no una identificación, al menos una equivalencia entre una pieza de

3. La fuente más antigua que tenemos de este mito es Hesíodo, Teogonía 453; 485-500.

4. DAVIDSON ofrece una interesante exposición sobre este tema (1995, 364-366).

5. VERNANT habla de la función de piedras «dobles» en la religión griega (1985, 325338) y asimismo sobre la figura de piedra vestida (ibidem, 65 y ss.) en relación con el culto.

6. Ovidio, Fastos V, 115; Higinio, Fábulas, 139.

7. Escolio a Eneida 3.104. 
madera y un niño, en una versión del mito del nacimiento del héroe Meleagro, al que las Moiras visitaron y dijeron que viviría tanto tiempo cuanto tardara en consumirse un tizón en el fuego ${ }^{8}$. La madre del niño, Altea, cogió el tizón y lo guardó, y, finalmente, Meleagro murió cuando Altea, muchos años después, arrojó el tizón al fuego.

En estas dos narraciones míticas un objeto inanimado se convierte en "doble» de una persona, un dios o un héroe, y puede identificarse con él hasta el punto de sustituirlo o asumir su personalidad. La relación de estas historias con la maternidad se muestra en el hecho de que la madre tiene siempre un papel especial, pues su intención es en ambos casos salvar al niño de ser destruido.

Otro texto clásico relevante es el pasaje del Satiricón de Petronio (LXIII, 1-12) en el que una madre que quiere coger en sus brazos el cuerpo de su hijo muerto es atacada por las éstriges (lat. strigae) y el hijo es arrebatado por estas y sustituido por una especie de muñeco de paja. Riccardo Castellana ve este pasaje como el primer testimonio literario del motivo del changeling (2014, 43-47) y anticipa algunos de los ejemplos que vamos a ver a continuación, con la diferencia de que el hijo sustituido aquí ya estaba muerto.

Pero el motivo de los niños sustituidos se asocia fundamentalmente a la mitología y al folklore celta y aparece frecuentemente en recopilaciones de mitos y leyendas de Irlanda, Gales, Escocia y -dentro de la Península Ibérica- del País Vasco y Asturias, aunque hay ejemplos también en otros lugares y países fuera de este ámbito. El término inglés por el que se le conoce es changeling, atestiguado ya en el siglo XVI, aunque también se encuentra documentado el término francés chanjon (Doulet 2003, 35).

En líneas generales se trata de una mujer sobrenatural (especialmente un hada pero también una lamia o una xana) ${ }^{10}$ que rapta a un niño humano y lo sustituye por uno propio o de su propia raza que normalmente se caracterizará por ser más feo, débil y enfermizo que el niño humano ${ }^{11}$. En otros casos, la sustitución puede hacerse incluso con una imagen de madera

8. Apolodoro, Biblioteca 1.8 .

9. Cf. también BetTini $(1998,299)$. Sobre las éstriges como figuras de la mitología clásica, $c f$. OlipHANT (1913).

10. Las lamias eran mujeres sobrenaturales de la mitología vasca, las xanas de la mitología asturiana (ÁLVAREZ PEÑa 2001, 11-17). Sobre la relación de estos seres, especialmente las lamias, con la mitología griega, $c f$. GONZÁlez SERRANO-AguirRe (1997, 221-224) y AguirRe CASTRO $(1994,316)$.

11. Motivos F321.1, F321.1.2, F321.1.4, F321.1.4.3 en Thompson (1955-1958). 
del niño (Briggs 2003, 255) ${ }^{12}$, lo que nos recuerda el ya mencionado caso de Meleagro, aunque el tizón de madera que asume la personalidad del héroe no sea necesariamente una figura con apariencia de niño ${ }^{13}$.

La secuencia narrativa, tal y como la analiza Jean-Michel Doulet (2003, 11), es la siguiente:

1. La sustitución, que ocurre en ausencia de la madre humana ${ }^{14}$.

2. La constatación, que viene dada sobre todo por el físico y otras características del niño: es feo, peludo, no habla o no crece.

3. El consejo de las vecinas.

4. La revelación. Lo más normal es que el niño feérico traicione su naturaleza por su glotonería o por su reacción ante una acción absurda que motiva su asombro, con expresiones como: "Yo que soy viejo nunca he visto cocinar en una cáscara de huevo...", "Soy un viejo diablo, pero desde que vivo no he visto...».

5. La restitución. Los testimonios ofrecen una serie de rituales que llevar a cabo para la recuperación del niño humano, incluso utilizando el fuego: si sobrevive, es el niño auténtico, si muere, es que no se ha concedido el cambio y el niño era aún el niño ajeno, supuestamente más débil ${ }^{15}$.

Es interesante señalar que en estos casos el niño feérico nunca es el fruto de la unión de una mujer sobrenatural con un mortal (Doulet 2003, 18). En la mitología clásica los hijos fruto de una unión de ese tipo son héroes fuertes y poderosos, con una particular relación con la madre sobrenatural, como Aquiles o Eneas (Aguirre 1996, 441). En los casos de changelings, el hada prefiere a un niño humano porque es mejor que el suyo, en contraste con lo que ocurre en la mitología clásica, donde la raza

12. Motivo F451.5.2.3.1 en Thompson (1955-1958).

13. Curiosamente en el cuento del ilustrador americano Maurice Sendak Outside Over There (1981) inspirado por los relatos folklóricos de changelings, un recién nacido es cambiado por una figura del niño de hielo ( $c f$. WARNER 2018, 7).

14. A veces puede ser de noche mientras la madre duerme (cf. CASTELLANA 2014, 47).

15. Una tradición asturiana relacionada con las xanas dice que la madre no debe amamantar al niño y entonces aparecerá la xana diciendo "Toma 'l to mocosín y dame 'l mío pelosín» (Álvarez PeÑa 2001, 16). Estos procedimientos, efectivamente, podían acabar con la vida del niño, en la creencia de que si no sobrevivía eso confirmaba su verdadera naturaleza (DOULET 2003, 88). Sobre los rituales de desenmascaramiento y restitución del niño $c f$. también CASTELLANa (2014, 61-72). 
sobrenatural o divina es siempre superior a la humana. Podríamos ver aquí una creencia de origen cristiano que asume que el niño feérico tiene que ver con el demonio y por lo tanto el niño humano siempre será mejor. Para la Iglesia la naturaleza demoníaca de los changelings era incontestable ${ }^{16}$. También se considera la creencia de que la raza de las hadas estaba en decadencia y necesitaba sangre fresca (Callejo Cabo 1995, 74).

Y ¿qué ocurre con el niño humano mientras tanto? Normalmente parece que viviría con las hadas siendo cuidado y alimentado por ellas. Este aspecto coincidiría con el caso de Zeus criado por las ninfas que hemos comentado antes. También en la mitología clásica el dios Hefesto fue criado por las Nereidas ${ }^{17}$ y el dios Dioniso por las ninfas de la montaña Nisa ${ }^{18}$, aunque en estos dos últimos casos no hay sustitución del niño. En ocasiones, en cambio, los niños raptados eran cautivos esclavos de las hadas o víctimas en un tributo que supuestamente las hadas debían pagar al diablo (Briggs 2003, 67). Lógicamente, si la restitución se producía de forma satisfactoria, todo acababa felizmente.

Estas historias reflejaban la necesidad de explicar de una forma mágica o sobrenatural la causa de muchas enfermedades infantiles (Schoon Eberly 1988, 58-59) o, incluso, la maternidad fuera del matrimonio. Aparecen siempre en conexión con la relación natural madre-hijo. Son, en definitiva, historias de maternidad, en las que los hombres quedan en un segundo plano. Asimismo, en algunos casos, llegaban a legitimar el infanticidio $^{19}$.

El motivo del changeling o la sustitución de niños aparece atestiguado no solo en crónicas medievales y recopilaciones de leyendas del siglo XIX (Briggs 2003, 67; Briggs 1967, 115), como la de Lady Wilde en Irlanda (The Ancient Legends of Ireland ${ }^{20}$, sino también en algunas obras literarias de las cuales la más significativa y célebre sería la que ya hemos citado: $E l$ sueño de una noche de verano de Shakespeare (1595), donde, mediada la

16. Doulet 2003, 96. Que un changeling era un hijo del diablo, sin alma, era también la visión de Lutero en relación con ciertas creencias populares en Alemania ( $c f$. AsHLiman 1997). Esta idea también está visualmente representada en una pintura de principios del siglo XV de Martino di Bartolomeo, La leyenda de San Esteban, en la que se ve que el diablo roba a un bebé y deja otro (con apariencia de diablo) en su lugar.

17. Homero, Ilíada 18, 394-405.

18. Himno Homérico a Dioniso 3-6.

19. En el mito griego una versión del nacimiento del dios Hefesto (Ilíada 18, 394-9) relata que, al ver Hera a su hijo tullido y deforme, lo arrojó del Olimpo. Por supuesto, el dios no murió, pero la intención de rechazo del recién nacido a causa de sus características físicas es la misma que en estas historias.

20. Publicado por primera vez en 1887. 
primera escena del acto II, Puck, el duendecillo asistente principal de Oberón el rey de las hadas, se refiere a un niño -originariamente un príncipe indio- al que denomina un changeling ${ }^{21}$.

For Oberon is passing fell and wrath, because that she as her attendant hath a lovely boy, stolen from an Indian King. She never had so sweet a changeling. (II. 1. 20-23) ${ }^{22}$

Celoso de la atención al niño, Oberón quiere que esté a su servicio, pero Titania rechaza esta petición adoptando al niño como suyo. En realidad, el niño cambiado figura en la obra simplemente como un objeto de deseo que suscita los celos de Oberón. Hay una inversión en el tema, puesto que aquí nos encontramos con lo que le ocurre a un niño humano robado que se encuentra en el país de las hadas con sus reyes, Oberón y Titania, que discuten sobre su custodia, en lugar de tener la perspectiva de los padres humanos. Es interesante aquí también el hecho de que la madre auténtica del niño humano era una especie de servidora de Titania, por lo que el niño no ha sido obtenido con violencia, sino por la amistad de Titania hacia ella. Titania compartía una relación de camaradería con ella y, al morir esta, se queda con el niño por su bien, asumiendo el papel de su madre ${ }^{23}$.

Hay aún otra mención al tema de los niños cambiados en Shakespeare, en una temática diferente que en este caso no tiene nada que ver con el mundo fantástico de las hadas: se trata de Cuento de invierno (1611), en donde, cuando un viejo pastor encuentra a un bebé, Perdita, la hija del rey de Sicilia abandonada al nacer por creerse que se trata del fruto de una relación ilícita, supone que es un changeling en la acepción de «niño dejado por las hadas":

21. Sobre esta escena y el personaje del changeling, cf. SLIGHTS (1988, 259-272). También en https://journeys.dartmouth.edu/exploratoryshakespeare/2015/07/07/changeling-usage-in-a-midsummer-nights-dream-vs-european-folklore/ [consultado el 14-7-2021].

22. "Oberón está muy enfurecido contra ella (Titania), porque lleva de paje a un hermoso doncel, robado a un monarca de la India. Jamás había poseído ella un objeto sustraído tan encantador». Traducción de Luis Astrana Marín. Hay que notar que en las traducciones al español de la obra este personaje aparece como "niño robado", "doncel robado", "objeto sustraído", un "paje", por lo que se pierde el sentido original de la palabra y su asociación con el mundo de las hadas. $C f$., por ejemplo, en la edición de Astrana Marín y en su prólogo de Vicente Molina Foix (Alianza Editorial, 2005).

23. De manera que el niño es más adoptado que robado: «His mother was a vot'ress of my order..." (II, i.123 y ss.); "And for her sake do I rear up her boy..." (260-261). 
So, let's see -it was told me I should be rich by the fairies. This is some changeling. (III. 3. 113-115) ${ }^{24}$

Estas referencias reflejan cómo este término pervivía en la época y era familiar para el público de Shakespeare ${ }^{25}$. En esa época, además, changeling también venía a significar en general «idiota» o "tonto», como se puede ver en la obra teatral de Thomas Middleton y William Rowley The Changeling (1622), en la idea de que se suponía que aquellos que eran mentalmente débiles o retrasados eran en realidad niños cambiados por las hadas. El título alude a los cambios que se operan en los personajes a lo largo de la obra, pero sobre todo a un personaje, Antonio, que es retrasado: "Tony, Tony, 'this enough and a very good name for a fool» (I. 2. 101) ${ }^{26}$.

Otro ejemplo literario de este motivo ya en el siglo XIX es el poema de W. B. Yeats The stolen child (escrito en 1886). Yeats, junto con Lady Wilde y Lady Gregory, fue el impulsor de la resurrección de los cuentos folklóricos irlandeses. No es de extrañar, pues, en este caso, su uso del tema dado el influjo del mundo de las hadas en su poesía ${ }^{27}$. En el poema las hadas no roban exactamente al niño (lo mismo que ocurre en el caso de El sueño de una noche de verano), sino que lo atraen para que vaya con ellas voluntariamente, ofreciéndole primero los frutos más rojos y exquisitos y luego tentándole con su mundo que sería mejor que el humano. El poema está escrito desde el punto de vista del hada que se lleva al niño y no hay ninguna alusión a la madre auténtica ni a la procedencia de este. El poema se puede entender, en definitiva, como una metáfora del regreso a la inocencia y a la infancia, pero también sobre el regreso al pasado y a las tradiciones irlandesas, ya que el poeta localiza claramente la acción en ciertos lugares de Irlanda que le eran familiares.

La trayectoria literaria de este tema no termina aquí. Incluso ya en época reciente, en los siglos Xx y xxI, tenemos ejemplos de obras con este motivo. Luigi Pirandello escribió Il figlio cambiato, un relato breve ${ }^{28}$ que muestra la expansión del tema a otros ámbitos geográficos y culturales, inspirado en una leyenda siciliana y ambientada en Sicilia a principios del

24. Sobre Cuento de invierno y el tema del changeling, cf. CASTELLANA (2014, 101-103).

25. La época de Shakespeare fue la gran época de poesía relacionada con las hadas en la literatura inglesa ( $c f$. BRIGGS 1967, 153-154). Sobre el tema de las hadas y Shakespeare, $c f$. en general LATHAM (1930) y WiLSON (1962. 184-220).

26. Cf. Three Jacobean Tragedies. The Penguin English Library, 1963, p. 36.

27. WARNER $(2018,10-11)$. Sobre la influencia de este motivo en otros autores irlandeses, $c f$. SHIMOKusu (2005).

28. Publicado en la colección Novelle per un anno en 1923. 
siglo $\mathrm{XIX}^{29}$. Su trama, contada por un narrador externo, parte de la situación de una madre, Sara, que, estando sola en casa, afirma que su bebé ha sido raptado y sustituido por otro. A la pregunta de las vecinas de ęquién lo ha robado?, la respuesta es «Le Donne», unas brujas, espíritus de la noche, en las que, obviamente, las mujeres creían. Las vecinas asimismo le confirman que el niño, efectivamente, tiene ahora una apariencia distinta: ya no es blanco y rubio, sino negro y feo. Estaba claro, pues, que estas brujas habían entrado en la casa y lo habían cambiado. Sin embargo, en ningún momento se plantea aquí la posibilidad de un sustituto sobrenatural, se asume siempre que el niño es otro niño humano, de otra madre, un niño físicamente inferior. Es interesante señalar cómo el narrador alude a que en realidad al niño le había sobrevenido una enfermedad durante la noche. Sara, al principio, no quiere atender a este niño, pero, finalmente, por consejo de una mujer, Vanna Scoma (de la que se decía que tenía tratos con las «Donne»), lo cuida como si fuera suyo, en la idea de que, si le trata bien, eso repercutirá en su hijo auténtico y un día podrá recuperarlo. La llegada de un nuevo bebé para Sara sugiere que ese niño representará de alguna manera la recuperación del otro, en una idea tradicionalmente asociada con madres que pierden trágicamente a un hijo y conciben otro para "sustituirlo". De manera que en esta historia encontramos, por un lado, las creencias supersticiosas en seres sobrenaturales (las "Donne») y la actitud de una madre que hace frente a la desgracia de un hijo enfermo o discapacitado y que, a través de esas creencias (fomentadas por Vanna Scoma en una especie de piadosa mentira), puede imaginar una sustitución de carácter mágico y a su verdadero hijo, sano y hermoso, creciendo en otro lugar.

Una popular autora contemporánea de novelas de misterio, Sophie Hannah, aborda el tema en Little Face (2006), con la historia de una madre que, de forma similar a la de Pirandello, afirma que su niña recién nacida no es su hija, supuestamente sustituida en su ausencia. La novela no explora suficientemente la cuestión de quién sería esa otra niña ni hay nada de sobrenatural en ella (y al final ni siquiera existe tal cambio), pero se plantean los problemas psicológicos relacionados con la maternidad: la madre que, tras el parto, se ve incapaz de cuidar al bebé o la asociación psicológica del bebé como parte de un marido no deseado. El motivo del changeling no tiene aquí un trasfondo mítico, religioso o supersticioso ni hay una razón para sospechar una enfermedad en la criatura, sino que se

29. Analizada detenidamente por Riccardo Castellana en su excelente libro (2014, 137-178). 
plantea en una trama de ficción criminal encaminada a desvelar al culpable de un asesinato.

Un medio artístico moderno en el que el tema del changeling ha cobrado una relevancia especial es el cine. De esta manera podemos ver cómo el tema va más allá de la literatura y aparece reelaborado actualmente en otro tipo de narrativa. Empezaré por dos películas, bien distintas en su trama argumental, que muestran a través de sus títulos un uso consciente y una intención de recordar al espectador este motivo ${ }^{30}$.

The Changeling (1980), dirigida por Peter Medak, es un film canadiense que figura dentro de la categoría de película de terror o, más concretamente, una ghost story, típica historia de casa encantada, en la que el niño cambiado -el auténtico y legítimo- revela su muerte a través de su presencia fantasmal en la casa donde vivió. Investigaciones llevadas a cabo por el protagonista descubren que un niño de seis años, inválido, fue asesinado por su padre, que temía que su hijo no alcanzara los 21 años, edad en la que debería heredar una enorme fortuna. Para asegurar la herencia, el padre reemplazó al niño muerto por un niño de un orfanato local y lo llevó a Europa con la pretensión de buscar tratamiento para su enfermedad. Después de varios años el padre regresó con el niño, entonces de 18 años, afirmando que se había recuperado de su enfermedad. El changeling, es decir, el niño impostor, es ahora un hombre mayor, un poderoso e influyente senador de los Estados Unidos y hombre de negocios.

Por lo tanto, tenemos en esta historia algunos detalles en relación con nuestro motivo: en primer lugar, la inversión de uno de los aspectos del tema del changeling: el niño cambiado es el niño sano que sobrevive, mientras que el auténtico y legítimo hijo de la familia es inválido y enfermo. Otro aspecto sería la ausencia de la madre, personaje importante en los relatos míticos, dado que los personajes de esta historia son masculinos: los dos niños intercambiados y el hombre testigo de las apariciones fantasmales que desvela lo ocurrido. Es el padre del niño el que lo mata y efectúa el cambio, de manera que el padre asume el papel de la madre-hada que sustituye a este niño legítimo por el falso. El mundo sobrenatural que representa el mundo de las hadas adonde el niño humano es llevado es aquí el mundo del más allá al que pertenece ahora el fantasma de Joseph. La presencia de unas medallas de bautizo, una que identifica al niño muerto y otra que está en posesión del senador, hace que este reconozca la verdad en los

30. Los títulos que estas dos películas recibieron en español (Al final de la escalera y El intercambio) demuestran que el nombre changeling y su relación con el motivo de los niños sustituidos no es familiar para un público hispanohablante. 
momentos anteriores a su trágica muerte en el incendio de la casa provocado por el fantasma. La medalla -como símbolo cristiano- nos recuerda los elementos de protección contra las hadas como seres paganos en las tradiciones con influencia cristiana y, asimismo, contradice la creencia de que los niños raptados eran los no bautizados, en la idea de que antes del bautismo el bebé era vulnerable a las hadas (Purkiss 2001, 59; Briggs 1967, 115).

En contraste, en la película Changeling, producida por Clint Eastwood en 2008 y protagonizada por Angelina Jolie, todo gira en torno a una madre y sus profundos sentimientos de dolor y frustración ante la desaparición de su hijo y, especialmente, ante la actitud de la policía que la obliga a aceptar a otro niño como el suyo. En 1928 Christine Collins vive sola con su hijo Walter, de nueve años. Un día regresa tarde después del trabajo y encuentra que su hijo ha desaparecido. Cinco meses más tarde el capitán J. J. Jones de la policía comunica a la señora Collins que han encontrado a su hijo y que viene en tren a reunirse con su madre. Sin embargo, ella no reconoce al niño como su hijo y, aunque el capitán Jones afirma que el niño puede haber cambiado en ese tiempo, la madre insiste en que no es su hijo. Pronto confirma que efectivamente no lo es y puede probarlo por razones físicas - por ejemplo, el hecho de que este niño está circuncidado y su hijo no, o que es unos centímetros más bajo que Walter-, pero la policía no acepta sus argumentos. Entretanto se descubre a un niño que ha escapado de un asesino en serie de menores y el caso toma una nueva dirección, asumiéndose que la desaparición de Walter podría estar conectada con este suceso, a la vez que el niño falso, el changeling, termina por admitir su verdadera personalidad.

Un aspecto en esta historia es el intenso amor de la madre hacia su hijo, que coincide con algunas de las tradiciones folklóricas de changelings en el sentido de que este amor sería la causa que motivaría el rapto y el cambio (Doulet 2003, 119-120). También coincide con la mayoría de los testimonios sobre este motivo el hecho de que es la madre la que trata por todos los medios de recuperar a Walter, poniéndose ella misma en contra de todas las autoridades y la policía, lo que la lleva incluso a ser tomada por loca.

Una interesante aproximación al tema que nos ocupa, el de una mujer que sospecha la sustitución de su hijo por otra criatura, en este caso, sobrenatural, es la irlandesa The Hole in the Ground (2018), un film de terror dirigido por Lee Cronin. El director usa conscientemente el folklore irlandés relativo al mundo de las hadas para convertir la historia en un relato de la ansiedad de una madre, personificando uno de los miedos más profundos de la mujer: el de perder a su hijo. De nuevo aquí se plantean los dos aspectos que hemos visto ya en las modernas recreaciones del tema: el 
folklore relativo al mundo de las hadas y la enfermedad mental ${ }^{31}$. La película nos presenta a una madre, Sarah, que se traslada a vivir en el campo con su hijo Chris. Se sugiere que anteriormente ha habido problemas con el padre del niño y una tormentosa separación que la llevó a tomar medicación, quizá para ansiedad o depresión, lo que indicaría cierta debilidad mental o emocional previa que luego podría explicar su conducta. Esto en ningún momento se plantea en la Christine Collins de Changeling. Un día el niño sale de casa y desaparece, pero cuando la madre llama a la policía el niño está de vuelta en su cuarto. Entonces empieza a mostrar detalles que contradicen su conducta habitual, por ejemplo, su apetito, pues ahora parece comer con glotonería cuando antes la madre se esforzaba por hacerle comer, y manifiesta otras actitudes inquietantes. Sarah entonces empieza a cuestionar la identidad de su hijo y a afirmar que ha sido sustituido por una criatura procedente de un misterioso y enorme agujero que hay en el bosque, que sugiere los llamados «agujeros de las hadas ${ }^{32}$, tradicionalmente entradas al mundo de las hadas, como muchos relatos de origen folklórico nos recuerdan.

Otro personaje interviene en esta película: una vecina, Noreen, que, según cuenta su marido, empezó a afirmar que su hijo de ocho años en realidad no era su hijo y acabó atropellándolo ella misma. Sarah aparece, pues, en paralelo con Noreen, de quien se supone que tenía una enfermedad mental, y el desarrollo de la película, aunque no está del todo claro, podría inducir a pensar en un final semejante para el hijo de Sarah. El hecho de que tras el final el director haya incluido una balada popular irlandesa, The Cruel Mother, sobre una mujer que comete infanticidio puede llevar a la idea de que se ha usado para sugerir cómo el tema del changeling en el folklore servía para justificar el infanticidio y deja en el aire la pregunta de si la enfermedad mental de Sarah la llevaría, como a Noreen, a atentar contra la vida de su hijo ${ }^{33}$. Sarah encuentra al niño auténtico en el agujero y se

31. https://fathersonholygore.com/2019/03/01/the-hole-in-the-ground-a-surrealnightmare-of-motherhood-and-mental-illness/ [consultado el 8-5-2020].

32. Un ejemplo literario de un agujero de las hadas o de la "gente menuda" aparece en el relato de Arthur Machen The Shining Pyramid, publicado por primera vez en 1895 e inspirado por el folklore galés (también de origen celta).

33. Esta balada se relaciona con la época de la gran hambruna en Irlanda cuando las madres que no podían alimentar a sus hijos recurrían al infanticidio. Como ya hemos comentado, la creencia en la naturaleza sobrenatural de un niño con ciertos patrones de apariencia y conducta, sospechoso de ser un changeling, podía llevar a acciones por parte de la madre que acabaran con la vida de la criatura. A menudo esos métodos de revelar la verdadera naturaleza del niño eran poco más que formas de infanticidio socialmente aceptadas (cf. SCHOON EBERLY 1988, 62). 
ve ella misma duplicada también en el mismo sitio y aquí nos encontramos con el motivo del doppelgänger o «doble»" ${ }^{34}$, con una confrontación entre el original y el usurpador. La película deja algunos cabos sueltos, pero en general resulta un buen ejemplo de cómo el motivo del changeling o la sustitución de un niño puede ser utilizado vinculado a estados mentales de una madre, de ansiedad en relación con sus hijos, en situaciones que se pueden interpretar como sobrenaturales o no.

Finalmente, voy a considerar una película de terror dirigida en 2016 por William Brent Bell, The Boy, que se acerca al tema del hijo sustituido de muy diferente forma, volviendo ahora a la sustitución del niño, no por otro niño, sino por un objeto inanimado, de la manera en que veíamos la piedra entregada a Crono por Rea en lugar de su hijo Zeus en el mito hesiódico de sucesión de los dioses. La trama del film es la siguiente: una joven americana, Greta, viaja a Inglaterra para trabajar de au pair del niño de una pareja madura en una elegante mansión en el campo. A su llegada los padres se disponen a presentarle al niño, llamado Brahms, que estará a su cuidado y para su sorpresa se encuentra con que se trata de un muñeco de porcelana al que ellos tratan como si fuera su hijo auténtico. Los padres se van a ausentar por lo que Greta tendrá que encargarse del supuesto niño y de la casa, dejándole una serie de reglas que tiene que seguir que incluyen leer al niño, ponerle música, prepararle la comida y acostarlo a las horas debidas.

La película juega con la posibilidad de que el muñeco tenga un carácter sobrenatural, pero cuando se desvela el misterio de lo que realmente ocurre en la casa se descubre que es el verdadero Brahms el que actúa, que está vivo y es ahora un joven que vive oculto en los muros de la mansión donde los propios padres lo escondieron desde que, cuando tenía ocho años, mató a una niña y provocó el incendio de la casa ${ }^{35}$. Por lo tanto, la sustitución la efectúan los padres, entre su hijo vivo que ha ido creciendo

34. Este enorme agujero se podría también asociar a los agujeros o círculos dejados por objetos extraterrestres en el cine de ciencia-ficción, por ejemplo, en La guerra de los mundos (1953), siguiendo la novela de H. G. Wells, o en la película Señales (Night Shyamalan, 2002); igualmente el tema de la duplicación de una persona nos recuerda la película de ciencia ficción The Invasion of the Body Snatchers (1956), cf. KOGEL-SCHAEFER (2011).

35. Esta figura del hombre de rostro deformado que vive escondido en las entrañas de un edificio nos recuerda la novela de Gaston Leroux El fantasma de la ópera (1909), con la historia adaptada en el musical de 1986 de Andrew Lloyd Webber y posteriormente en la película dirigida en 2004 por Joel Schumacher. Incluso parece inspirarse en las versiones más modernas de la historia de El fantasma de la ópera en otro detalle: cuando la máscara que lleva el verdadero Brahms y que es idéntica a la cara del muñeco se rompe, revela parte quemada de su rostro. 
y convirtiéndose en un hombre y el muñeco que permanece en la edad en que supuestamente murió. El auténtico Brahms, un hombre violento como ya lo era en su infancia, había estado vigilando a Greta y, cuando se da a conocer, después de atacarla, le pide -con la voz de un niño pequeño- que se quede con él, aunque ella finalmente logra escapar de la casa.

El muñeco, por lo tanto, se propone como imagen de la persona viva. Si en el mundo infantil los muñecos están para que sus dueñas (o dueños) los imaginen con vida e interactúen con ellos (Bettini 2015, 28), aquí son los adultos -los padres del niño/muñeco- los que se comportan como si tuviera vida y fuera el auténtico hijo y, además, exigen de la niñera la misma actitud que, lógicamente, a Greta le parece primero una broma y luego fruto de una mente perturbada. Los muñecos con vida o que cobran vida son un tema bien conocido en la literatura infantil (por ejemplo, Pinocho de Carlo Collodi, 1883) y asimismo en la literatura y el cine fantástico o de terror $^{36}$. Se trata una vez más del motivo del doppelgänger o el "doble ${ }^{37}$.

Hay dos detalles en esta película que aluden a la maternidad y la relación entre padres e hijos: una de las razones por las que Greta había dejado su país era porque había estado embarazada y había abortado como resultado de una relación con un hombre violento, Cole, que luego se va a presentar en la mansión inglesa para exigir a Greta que vuelva con él. De manera que Greta aparece, en cierto modo, como una madre frustrada que pasa a dedicar sus cuidados maternales de un hijo propio (el que hubiera tenido) a un muñeco. El muñeco Brahms aparecería entonces como un changeling del hijo no nacido de la propia Greta. Por otro lado, los padres de Brahms, especialmente la madre, van a renunciar al hijo (al Brahms vivo y al muñeco) y se desvela la verdadera razón de contratar a Greta: dejarla para siempre encargada de la casa y de la situación, ya que el propósito de su viaje no es irse de vacaciones -como anunciaron- sino suicidarse. Esto implica que la carga emocional de lo que ocurre con Brahms resulta

36. Los muñecos, infantiles o de ventrílocuos, se han convertido en un motivo generalizado del cine de terror, aunque en la mayoría de los casos no aparezca el tema de la sustitución como en el ejemplo que estamos tratando, sino más bien la idea de que albergan algún tipo de espíritu malvado, lo que produce la desaparición de la barrera entre lo animado y lo inanimado. Algunos ejemplos bien conocidos serían Dolls (1987), El muñeco diabólico (1988), Annabelle (2014) (con secuelas posteriores) o, incluso, la decepcionante Brabms: The Boy 2 (2020). Por otro lado, los muñecos, en especial las muñecas, juegan un papel importante en las obras de numerosos escritores victorianos, fundamentalmente como juguetes para niñas, siempre como objetos inanimados que invitan a la interacción (cf. GONZÁLEZ-POSSE 2012).

37. Algunos ejemplos en Cavaliero $(1995,111)$. Según Jones $(2018,117)$ muchas de las grandes obras de terror del siglo XIX son cuentos de «doble». 
ya insoportable para ellos y han tenido que tomar tan drástica decisión. Se intuye por tanto que, a lo largo de los años, han interactuado con su propio hijo -quien, aparentemente, ejercía un total dominio sobre sus padres- a través del muñeco, al que por otra parte conservan como un niño lleno de buenas cualidades: limpio, aseado, bien educado, que disfruta con la música y la lectura, en contraste con el verdadero hijo, el psicópata asesino oculto en la casa.

\section{CONCLUSIONES}

En definitiva, el tema del hijo sustituido refleja una serie de situaciones y conflictos relacionados con la maternidad ${ }^{38}$. En los ejemplos del mito clásico y el folklore se puede ver un deseo por parte de una madre de preservar a su hijo, recuperar a un hijo perdido, devolverle a una situación de reconocimiento de sus derechos que le han sido negados o -en el caso de que se trate de las madres hadas, lamias o xanas- mejorar la descendencia a través de una progenie de mejor calidad (en cuanto a salud y fortaleza) y para ello efectúa la sustitución de un hijo propio inferior por otro superior $^{39}$. La creencia en la naturaleza sobrenatural de un niño con un defecto congénito era ya frecuente en la Antigüedad y luego en la Edad Media.

Por otro lado, el tema que subyace en muchos de los casos es el de la mortalidad infantil en la época en la que los relatos de changelings circularon: estas creencias ayudaban a asumir mejor la tragedia de la pérdida de un hijo y, a la vez, las madres que torturaban o abandonaban a un hijo enfermo o más débil se justificaban aduciendo que se trataba de un changeling y no de su propio hijo, que había sido robado.

Los ejemplos modernos dejan ver los conflictos de ansiedad y depresión relacionados con la maternidad: la actitud de una madre que tiene que enfrentarse a un hijo mental o físicamente enfermo, y el miedo fundamental de cualquier madre de perder a su hijo o el dolor por haberlo

38. De forma similar el mito clásico de Lamia, la mujer que roba niños para devorarlos, refleja los miedos de la mujer en relación con la maternidad ( $c f$. GONZÁLEZ-RIVAs 2018).

39. Marina WARNER $(2018,94)$ recuerda la teoría de Freud tradicionalmente llamada "family romance» según la cual los niños fantasean con la idea de que sus padres son impostores y que ellos han sido robados de una familia mejor, más amable y más rica (cf. también BetTelheim 1979, 67-69). Esto sería una inversión del tema de la sustitución, vista desde la perspectiva del hijo y no de la madre (o padres). 
perdido. En algunos casos extremos esa situación emocional podría llevar a la enajenación mental.

Hoy en día las consideraciones sociales que afectaban a las personas, especialmente a los niños, más débiles o con discapacidad intelectual y que hacían que se les tomara por changelings explicando de esta forma sus carencias ya no se dan. Sin embargo, la literatura o el cine recuerdan de nuevo estos motivos cuando ciertos temas de violencia o abuso infantil y, en definitiva, la situación del niño como un ser indefenso se sitúan en primera línea de actualidad. La literatura y el cine han revisado y reutilizado los antiguos mitos para reflejar una realidad de conducta humana que es válida hoy como lo fue en la época en que las antiguas historias se transmitieron. Estas historias han sido pretexto para una narración de muchos tipos: fantástica, de horror o de misterio, pero, en definitiva, en su mayoría se trata de plasmar la angustia de una madre que se enfrenta a un hijo diferente o a la pérdida de su hijo. Como afirma Castellana (2014, 161) el tema del hijo sustituido como motivo literario (y, podríamos decir, también, cinematográfico) revela el espíritu de cada época y se abre a la reinterpretación de cada autor. La presencia en nuestra cultura popular de motivos y arquetipos pertenecientes a los antiguos mitos y leyendas atestigua la vitalidad de nuestra tradición cultural y las conexiones entre las sociedades del pasado y la nuestra ${ }^{40}$.

\section{BiBLIOGRAFÍA}

Aguirre CAstro, Mercedes. "El tema de la mujer fatal en la Odisea». Cuadernos de Filología Clásica(egi), 1994, 4, pp. 301-317.

AguiRre CASTRO, Mercedes. "Casarse con una hechicera: mito griego y tradición folklórica europea». Epos, 1996, 12, pp. 435-442.

Álvarez PeÑa, Alberto. Mitología asturiana. Gijón: Pieu Urriellu, 2001.

Ashliman, Dan L. "Changelings: an Essay", 1997 http://www.pitt.edu/ dash/changeling.html [consultado 8-5-2020].

BetTelHeim, Bruno. The Uses of Enchantment: The Meaning and Importance of Fairy Tales. London: Thames\&Hudson, 1979.

BetTini, Maurizio. Nascere: storie di donne, donnole, madri ed eroi. Torino: Einaudi, 1998.

BetTini, Maurizio. Muñeca. Madrid: Casimiro, 2015.

40. Sobre la convergencia entre film y folklore, $c f$. SHERMAN-KOREN (2007); sobre mito clásico y cine en general, $c f$. WINKLER (2001). 
EL HIJO SUSTITUIDO: PROYECCIONES DE UN MOTIVO MÍTICO Y FOLKLÓRICO EN LA LITERATURA...

BRIGGS, Katherine. The Fairies in Tradition and Literature. London: Routledge\&Kegan Paul, 1967.

Briggs, Katherine. Diccionario de las hadas. Madrid: José J. de Olañeta, 2003.

Callejo Cabo, Jesús. Hadas. Guía de los seres mágicos de España. Madrid: Edaf, 1995.

Castellana, Riccardo. Stori di figli cambiati. Fate, demoni e sostituzioni magiche tra folklore e letteratura. Pisa: Pacini Editori, 2014.

CAVAliERO, Glen. The Supernatural and English Fiction. Oxford: Oxford University Press, 1995.

Davidson, John. "Zeus and the Stone Substitute». Hermes, 1995, 123, pp. 363-369.

DOulET, Jean-Michel. Quand les démons enlevaient les enfants. Les changelings: étude d'une figure mythique. Paris: Presses de l'Université Paris-Sorbonne, 2003

GonZÁLEz-Posse, Maria Eugenia. Galatea's Daughters. Dolls, Female Identity and the Material Imagination in Victorian Literature and Culture (Dissertation). The Ohio State University, 2012.

GonZÁlEZ-RIVAs FernándeZ, Ana. "Reconstructing the Myth of Lamia in Modern Fiction: Stories of Motherhood, Miscarriage and Vengeance». The International Journal of the Humanities, 2018, 16:1, pp. 25-38.

GONZÁlez-SERRANO, Pilar y Mercedes AGuirRe. "Espíritus malignos, dragones y lamias». Más cerca de Grecia, 1997, 12, pp. 213-224.

Jones, Darryl. Sleeping with the lights on. The Unsettling Story of Horror. Oxford: Oxford University Press, 2018.

Kogel, Dennis y Iris SchaefER. "The Doppelgänger motif in Science-Fiction film». En Georgi, S. y K. Loock (eds.). Of Body Snatchers and Cyberpunks: Student Essays on American Science-Fiction. Universität Göttingen, 2011, pp. 125-141.

LATHAM, Minor White. The Elizabethan Fairies: The Fairies of Folklore and the Fairies of Shakespeare. Columbia University Press, 1930.

Oliphant, Samuel Grant. "The Story of the Strix: Ancient». Transactions and Proceedings of the American Philological Association, 1913, vol. 44, pp. 133-149.

Purkiss, Diane. Troublesome Things: A History of Fairies and Fairy Stories. London: Penguin, 2001.

SCHOON EBERLy, Susan, "Fairies and the Folklore of Disability: Changelings, Hybrids and the Solitary Fairy». Folklore, 1988, 99:1, pp. 58-77.

SHERman, Sharon R. y Mikel J. Koven. Foklore/cinema: Popular Film as Vernacular Culture. Logan: Utah State University Press, 2007.

SHIMOKusu, Masaya. Fairy Ireland. A Literary History of "Changelings". Tokyo: Heibon-sha, 2005.

SLights, William E. "The Changeling in A Dream». Studies in English Literature, 1500-1900, 1988, vol. 18, n. 2 (Spring), pp. 259-272.

Thompson, Stith. Motif-Index of Folk-Literature, 6 vols. Bloomington: IN, 1955-1958. Vernant, Jean Pierre. Mythe et pensée chez les Grecs II. París: F. Maspero, 1965.

Versnel, Henk S. "Greek Myth and Ritual: The Case of Kronos». En Bremmer, Jan (ed.). Interpretations of Greek Mythology. Kent, 1985. 
180

MERCEDES AGUIRRE CASTRO

EL HIJO SUSTITUIDO: PROYECCIONES DE UN MOTIVO MÍTICO Y FOLKLÓRICO EN LA LITERATURA...

WARner, Marina. Fairy Tale. A Very Short Introduction. Oxford: Oxford University Press, 2018.

Wilson, John Dover. Shakespeare's Happy Comedies. London: Faber, 1962.

Winkler, Martin M. Classical Myth and Culture in the Cinema. Oxford: Oxford University Press, 2001. 